\section{(6) OPEN ACCESS}

${ }^{1}$ Clinical Operational Research Unit, University College London, London, UK ${ }^{2}$ Clinic for Thoracic Surgery, Institute for Pulmonary Diseases of Vojvodina, University of Novi Sad, Novi Sad, Serbia

${ }^{3}$ Department of Cardiothoracic Surgery, National Heart and Lung Institute, Imperial College London, London, UK ${ }^{4}$ Wales Cancer Trials Unit, Cardiff University, Cardiff, UK

\section{Correspondence to} Professor Tom Treasure, Clinical Operational Research Unit, University College London, 4 Taviton Street, London WC1H OBT, UK; tom.treasure@gmail.com

Received 11 December 2013 Accepted 11 December 2013 Published Online First 10 January 2014

\title{
Pulmonary metastasectomy: what is the practice and where is the evidence for effectiveness?
}

\author{
Tom Treasure, ${ }^{1}$ Mišel Milošević, ${ }^{2}$ Francesca Fiorentino, ${ }^{3}$ Fergus Macbeth ${ }^{4}$
}

\section{ABSTRACT}

Pulmonary metastasectomy is a commonly performed operation and is tending to increase as part of a concept of personalised treatment for advanced cancer. There have been no randomised trials; belief in effectiveness of metastasectomy is based on registry data and surgical follow-up studies. These retrospective series are comprised predominately of solitary or few metastases with primary resection to metastasectomy intervals longer than $2-3$ years. Five-year survival rates of $30-50 \%$ are recorded, but as case selection is based on favourable prognostic features, an apparent association between metastasectomy and survival cannot be interpreted as causation. Cancers for which lung metastasectomy is used are considered in four pathological groups. In nonseminomatous germ cell tumour, for which chemotherapy is highly effective, excision of residual pulmonary disease guides future treatment and in particular allows an informed decisions as to further chemotherapy. Sarcoma metastasises predominately to lung and pulmonary metastasectomy for both bone and soft tissues sarcoma is routinely considered as a treatment option but without randomised data. The commonest circumstance for lung and liver metastasectomy is colorectal cancer. Repeated resections and ablations are commonplace but without evidence of effectiveness for either. For melanoma, results are particularly poor, but lung metastases are resected when no other treatment options are available. In this review, the available evidence is considered and the conclusion reached is that in the absence of randomised trials there is uncertainty about effectiveness. A randomised controlled trial, Pulmonary Metastasectomy in Colorectal Cancer (PulMiCC), is in progress and randomised trials in sarcoma seem warranted.

\section{INTRODUCTION}

Apart from resection for lung cancer, metastasectomy is the most frequent major operation undertaken in thoracic surgical departments. Metastasectomy represents $15-50 \%$ of the workload in European thoracic surgical departments.

The aim of this paper is to

A. review the nature the evidence used in support of pulmonary metastasectomy

B. reach some conclusion as to when, if at all, pulmonary metastasectomy is justified

C. explore the inherent risks of using observational reports as evidence for effectiveness.

Most patients having lung metastasectomy have had a primary cancer treated, and metastases are detected on routine surveillance, usually with whole body CT scans. Some metastases are discovered in the chest clinic in the course of the investigation of symptoms, or as chance X-ray findings, but most referrals for metastasectomy come from oncologists.

Lung metastases are generally asymptomatic. Occasionally metastases infiltrate the thoracic wall causing pain, contain a large volume of necrotic tumour or cause recurrent haemoptysis or retention pneumonia and are then resected for palliation.

\section{Background from the International Registry of Lung Metastasectomy 1997}

After the liver, lung is the next most common site of metastases. An important landmark publication is the 1997 report from the International Registry of Lung Metastasectomy (IRLM) of 5206 patients from Europe and North America. ${ }^{1} 2$

The authors report the data in four groups based on the primary cancer: germ cell tumour (7\%), melanoma (6\%), sarcoma (42\%) and epithelial tumours (44\%). There is a consistent finding in the registry and in institutional reports: irrespective of the cancer type, the fewer the metastases and the longer the interval before their appearance, the longer is survival after metastasectomy. In the registry, $46 \%$ of patients had a solitary metastasectomy and in $32 \%$ the interval since primary resection was more than 3 years. These are consistently favourable features for survival. The $18 \%$ with both features had a median survival of 61 months while for those with neither, median survival was 14 months. There was a wide difference in 5-year survival, depending on the type of cancer: $68 \%$ for germ cell, $37 \%$ for epithelial cancer, $31 \%$ for sarcoma and $21 \%$ for melanoma.

In current practice, colorectal cancer (CRC) is the commonest epithelial carcinoma for which pulmonary metastasectomy is advocated. For breast and lung cancer, pulmonary metastasectomy is rarely considered. Gynaecological, urological and upper gastrointestinal cancers appear in mixed series. Thyroid and kidney cancers may have isolated and slow growing metastases, but the number of cases is few so that we will not deal with them individually in this review. The general concerns about the nature of the evidence apply.

\section{OLIGOMETASTATIC DISEASE}

In $1995,{ }^{3}$ it was proposed that a clinical state of oligometastases existed 'amenable to a curative therapeutic strategy'. The oligometastatic state is generally limited to five or fewer metastases, ${ }^{4}$ which may be in more than one organ. ${ }^{5}$ A review of the subject suggests that this is neither a biological entity nor based on a mathematical analysis of metastatic frequency distributions, but rather that five is sufficiently few to be treated by ablative techniques. ${ }^{3}$ 


\section{ROLE OF TUMOUR MARKERS IN THE SELECTION OF PATIENTS}

Human chorionic gonadotropin (hCG) and $\alpha$ fetoprotein are used in non-seminomatous germ cell tumour (NSGCT) in order to detect persistent or recurrent tumour. ${ }^{6} 7$

An elevated carcinoembryonic antigen (CEA) of more than $5 \mu \mathrm{g} / \mathrm{L}$ is characteristic of CRC but may also occur in mesothelioma and a number of other tumours. It is raised in $3 \%, 25 \%$, $45 \%$ and $65 \%$ of Dukes' A, B, C and D, respectively. ${ }^{6}$ CEA falls after curative resection, and its subsequent elevation is particularly associated with liver metastases. ${ }^{6}$ An elevated CEA is associated with poor outcome after pulmonary metastasectomy for patients with $\mathrm{CRC}^{8}{ }^{8}$ which has led to the paradoxical situation where CEA elevation may trigger investigations that lead to referral for pulmonary metastasectomy while the same blood test is later a reason to decide against metastasectomy. ${ }^{9}$ Investigation and surgery triggered by CEA elevation have been shown to not improve survival. ${ }^{10}$

Very large numbers of tumour marker assays are ordered, many inappropriately, at high cost in themselves, and leading to further investigations that may be of no benefit. ${ }^{6}$

\section{GERM CELL TUMOURS}

NSGCT are the commonest cancers in young males. NSGCT is highly responsive to chemotherapy, and $90 \%$ can be cured. Of those with stage I disease, $8 \%$ develop pulmonary metastases. In the IRLM data, the survival graphs for NSGCT show a marked flattening after 2-3 years corresponding to the high probability of cure. ${ }^{1}$ After chemotherapy, there may be residual masses in the lungs, which may contain necrotic tissue or residual malignancy. In one-third of cases where there is malignancy, its histology differs from the primary cancer; surgical resection is thus useful in that it helps to guide further treatment with chemotherapy. ${ }^{11}$

Lung metastasectomy for NSGCT was reviewed as part of the European Society of Thoracic Surgeons (ESTS) Lung Metastasectomy Project. ${ }^{11}$ The literature was searched from 1998 to 2009 , and reports for analysis were those with 50 or more patients, operated on no earlier than 1980. This provided six studies including 740 patients of average age 27 years. Reported 5 -year survival was from $73 \%$ to $94 \%$ following pulmonary metastasectomy.

Multiple metastases and the finding of residual malignancy were associated with progression and death. Finding necrosis with no active disease defines patients who can be confidently spared chemotherapy. If necrosis only is found on one side, the same was found in the contralateral lung in 19 out of $20(95 \%)$ of cases (95\%; CI $75.13 \%$ to $99.87 \%$ ). This may spare an operation on the contralateral side. ${ }^{12}$ Long-term survival is not attributable to the surgery, but surgery is an established part of the management strategy for cogent evidence-based reasons.

Current indications for resection of lung metastases in these patients are

1. absence of response to chemotherapy

2. partial response followed by recurrence while on chemotherapy

3. recurrence after standard and second-line chemotherapy

4. to determine whether residual viable tumour is present

5. to resect enlarging benign teratomatous elements of the tumour. $^{11}$

\section{SARCOMA}

A systematic review found 15 reports of patients having a first pulmonary metastasectomy: five bone sarcoma, six soft tissue sarcoma and four mixed series. ${ }^{13}$ Sarcoma includes a wide range of pathological entities, but no clear distinction is drawn in management in published series of pulmonary metastasectomy, and for the limited scope of this review we will consider them together.

Metastases from sarcoma may be confined to the lung, and metastasectomy is a well-established component of the management of sarcoma. The Cooperative Osteosarcoma Study Group found that, of 202 patients who had metastases at diagnosis, $81 \%$ had lung metastases and $62 \%$ had only lung metastases. ${ }^{14}$ In an analysis of three European Osteosarcoma Intergroup (EOI) randomised controlled trials (RCTs) of chemotherapy, of 564 patients who had recurrence, 307 (54\%) had only lung metastases. ${ }^{15}$ In 2006, the UK National Institute of Health and Care Excellence (NICE), which issues guidance to the National Health Service in England and Wales, published a manual for commissioners of cancer service on 'Improving Outcomes for People with Sarcoma'. ${ }^{16}$ The manual, more about organisation than guidance on clinical practice, requires that a sarcoma multidisciplinary team should include a thoracic surgeon.

The systematic review included 18 follow-up studies reporting the selection and outcome for pulmonary metastasectomy in publication from 1991 to 2010. A first metastasectomy operation was reported in 1196 patients. Of 1357 patients, 43\% had a subsequent metastasectomy, some patients having 10 or more thoracotomies. Three studies were confined to patients having repeated pulmonary metastasectomy. The outcome measure was survival to 5 years. No RCTs or other forms of comparative analysis were found. No symptomatic or quality-of-life data were reported.

For bone and soft tissues sarcoma, respectively 34\% and 25\% of patients were alive 5 years after a first metastasectomy operation. Several series reported multivariate analyses. Survival was better with fewer metastases and longer intervals between diagnosis and the appearance of metastases. In the Thames Cancer Registry for 1995-2004, 5-year survival rates for all patients with metastatic sarcoma were $25 \%$ for bone and $15 \%$ for soft tissue sarcoma. Given that pulmonary metastasectomy is used in a highly selected favourable minority, it suggests that the benefit from metastasectomy cannot be large. Although it is a 'standard' component of sarcoma care, the evidence for benefit is weak. ${ }^{17}$

\section{MELANOMA}

Lung metastasectomy for melanoma was reviewed as part of the ESTS Lung Metastasectomy Project. ${ }^{18}{ }^{19}$ Two publications included registry data. There were 6129 patients in 22 years from the John Wayne Cancer Institute (JWCI) in 1971-1993 20 and 14057 patients from Duke University 1970-2004, spanning an additional decade. ${ }^{21}$ These data allow us to get some numerical perspective ${ }^{22}$ on the use of pulmonary metastasectomy in melanoma in the USA. The recorded incidence of at least one pulmonary metastasis was $16 \%$ (984/6129) and $12 \%$ (1720/14 057) (table 1). Of those with metastases, 11\% and $18 \%$ had pulmonary metastasectomy, ${ }^{20} 21$ a difference that probably reflects the increased interest in performing metastasectomy during the additional time frame. ${ }^{23} \mathrm{~A}$ subsequent report from JWCI in 45 patients, in whom tumour doubling times had been calculated on plain chest X-rays, showed that shorter doubling times were associated with poorer survival, which points to the biology of the cancer being the important determinant irrespective of the effect of metastasectomy. ${ }^{24}$ Immunotherapy has been proposed to limit microscopic melanoma, leaving macroscopic pulmonary metastases amenable to resection. $^{25}$ 
Table 1 Data on pulmonary metastases with melanoma, pulmonary metastasectomy and survival rates

\begin{tabular}{llllllll}
\hline Source & Dates & Melanoma cases & Pulmonary metastases & Pulmonary metastasectomy & Operation rate & 5-year survival & 10-year survival \\
\hline JWCI $^{20}$ & $1971-1993$ & 6129 & $984(16 \%)$ & 106 & $11 \%$ & $27 \%$ & \\
IRLM $^{1}$ & $1945-1995$ & Not known & Not known & 328 & & $22 \%$ & $16 \%$ \\
Duke $^{21}$ & $1970-2004$ & 14057 & $1720(12 \%)$ & 318 & $18 \%$ & Not clear & \\
\hline
\end{tabular}

IRLM, International Registry of Lung Metastasectomy; JWCI, John Wayne Cancer Institute.

The authors of the ESTS review suggested extremely restrictive conditions for metastasectomy in melanoma, ${ }^{19}$ and the overall summary of the ESTS leaders was that no recommendations could be made on the basis of this evidence. ${ }^{18}$

\section{COLORECTAL CANCER}

There have been about 100 reports of metastasectomy for CRC going back over 50 years but no randomised studies. ${ }^{26}$ The most comprehensive information on current practice is from a recent Spanish registry report of 543 patients. ${ }^{27}$ A national registry set up in Spain by Grupo Español de Cirugía Metástasis Pulmonares de Carcinoma Colo-Rectal (GECMP-CCR) collected data from 32 Spanish thoracic units on all patients with one or more histologically proven lung metastasis removed by surgery between March 2008 and February 2010. There were $65 \%$ men aged $32-88$ (mean 65 ) years. In $55 \%$ of patients, there was a solitary metastasis. The median interval between the primary cancer resection and metastasectomy was 28 months and serum CEA was low/normal in the majority. Liver metastases were present in $29 \%$ of patients at some point before pulmonary metastasectomy. A comprehensive review of practice since the mid-1960s shows how the case mix has changed relatively little over time. ${ }^{23}$ The mean age has increased from 60 to 65 years, and the proportion of patients with a solitary metastasis has gone down from $60 \%$ to $55 \%$. The proportion of patients who have had a prior liver metastasectomy has however risen markedly.

A recent systematic review and meta-analysis ${ }^{8}$ has considered outcomes for pulmonary metastasectomy in CRC. Twenty-five studies including a total of 2925 patients are included in this analysis. Overall 5-year survival after complete resection of pulmonary metastases ranged from $27 \%$ to $68 \%$. Outlying figures tend to be for smaller series. ${ }^{23}$ Three prognostic factors were associated with poor survival: (i) a short disease-free interval between primary tumour resection and development of lung metastases (HR 1.59, 95\% CI 1.27 to 1.98); (ii) multiple metastases (HR 2.04, 95\% CI 1.72 to 2.41); and (iii) elevated prethoracotomy CEA (HR 1.91, 95\% CI 1.57 to 2.32). Positive hilar and/or mediastinal lymph nodes (HR 1.65, 95\% CI 1.35 to 2.02) were predictive of poorer outcome, whereas a history of resected liver metastases (HR 1.22, 95\% CI 0.91 to 1.64) was not significant. ${ }^{8}$

\section{SHORTCOMINGS OF THE EVIDENCE FOR EFFECTIVENESS OF METASTASECTOMY}

The published evidence on pulmonary metastasectomy consists almost entirely of follow-up studies that are inevitably liable to a number of serious biases. ${ }^{28}$ A limitation in the IRLM data was the absence of a denominator. This weakness is central to much of surgical follow-up: only data on patients who have the completed treatment are retrieved.

The most important limitation is selection bias. Only 2-3\% of patients with pulmonary metastases, that is, 1 in 30-50 patients, have metastasectomy. ${ }^{27}$ Selected patients have favourable features: solitary or a low number of metastases and long interoperative interval, and in CRCs, non-elevated CEA. These are general prognostic features that will be associated with survival for all treatments and none. There is a common failure in reports of metastasectomy to distinguish between prognostic and predictive characteristics. ${ }^{29}$ Patients with favourable prognostic factors are likely to survive longer irrespective of treatment. ${ }^{29}$ Predictive factors are those that discriminate between patients likely and unlikely to benefit from particular treatments.

Negative predictive features include (i) an uncontrolled primary cancer, (ii) metastases at other sites, (iii) inability to achieve microscopically clear margins (R1/2) and (iv) mediastinal lymphatic spread. The first two are regarded by surgeons as reasons for exclusion. Surgeons strive to avoid the third and fourth because the metastasectomy fails in its intent to clear cancer. ${ }^{30}$ In more recent studies, failure to achieve R0 resection is commonly used to exclude patients from published reports. ${ }^{27} 3132$

In addition to these known prognostic and predictive features, patients are selected by successive doctors and multidisciplinary teams on numerous unspecified clinical criteria. The data for multivariate analyses are captured and entered at one point in time, whereas clinicians see patients two, three or more times as they undergo a series of tests. Experienced clinicians are able to identify patients likely to not do well: they know how to pick winners. The flat trajectory of indolent disease is readily separated from the clinical decline and radiological progression of patients with more aggressive malignant disease. This highly effective selection process is not captured in database, registry or follow-up studies.

In the absence of formal control groups, clinicians observe that the outcome for patients who have had metastasectomy is better than those with stage IV cancer. But patients having metastasectomy were generally free of evident metastases at the time of primary resection, and their metastases appeared on average 2-3 years later. They should not be compared solely with patient with metastases from the outset but with survival for patients with a similar stage mix at presentation or at primary surgery. ${ }^{33}$

There is a commonly made implicit assumption that all patients with metastases have a short prognosis. In a small study from over 30 years ago, 12 patients who had met the criteria for pulmonary metastasectomy, but in whom the operation was not carried out, were identified from the respiratory physicians' clinical records. Of the 12,3 were still alive 5 years later $(25 \%$; $95 \%$ CI $5.49 \%$ to $57.19 \%$ ). The point Åberg made was that it has been assumed, implied or claimed that the 5-year survival without operation is nil'. ${ }^{34}$ That was not the case. Nor is it the case in cancer registry records of large numbers of patients with metastases from sarcoma ${ }^{13}$ and CRC. ${ }^{33} 35$

Often response to chemotherapy, or non-progression, is required before metastasectomy, or repeat metastasectomy, is advocated. Among such patients it is not possible to separate the effect of any particular intervention, but they appear in 
metastasectomy series as if their survival was attributable to that operation. Being selected for surgery is often conditional on non-progression on chemotherapy. The patient needs to be alive to undergo metastasectomy and this may be the reason for the apparent association rather than because survival is a result of the metastasectomy itself. This is one of various manifestations of Immortal Time Bias. ${ }^{36} 37$ It is commonly claimed that second and third metastasectomy operations restore $\mathrm{control}^{38}$ or 'reset the oncological clock'. The diminishing denominator is overlooked. Patients still alive after a second or third treatment qualify to be in a cohort of patients having multiple treatments as a result of being alive, rather than being alive as result of the treatment.

\section{CONCLUSIONS}

In spite of abundant follow-up studies, the evidence for pulmonary metastasectomy is weak. There are no randomised trials and there is an absence of comparative analysis. The practice is driven by the feeling of a need to 'do something', most evident in repeated lung resections for sarcoma in the young. ${ }^{39}$ An association between survival and metastasectomy may be perceived, but lung metastasectomy fails the Bradford Hill tests of causality. ${ }^{40}$ Given the heterogeneity of the disease and in its rate of progression, and the multiplicity and repetition of treatments, it is not possible to distinguish the signal from the noise. ${ }^{41}$

There is sufficient uncertainty about the effectiveness of metastasectomy to fully justify RCTs. By far the commonest indication for metastasectomy is in CRC and so the Pulmonary Metastasectomy in Colorectal Cancer (PulMiCC) trial is confined to that disease and is recruiting at present.

Contributors All authors contributed to the manuscript. FF has worked on the systematic reviews and other studies that underpin this work. MM has been involved in the PulMiCC trial. FM is in the steering committee of PulMiCC. We have shared ideas about the material, and all authors have contributed to consecutive drafts and approved the final draft.

\section{Competing interests None.}

Provenance and peer review Commissioned; internally peer reviewed.

Open Access This is an Open Access article distributed in accordance with the Creative Commons Attribution Non Commercial (CC BY-NC 3.0) license, which permits others to distribute, remix, adapt, build upon this work non-commercially, and license their derivative works on different terms, provided the original work is properly cited and the use is non-commercial. See: http://creativecommons.org/ licenses/by-nc/3.0/

\section{REFERENCES}

1 Pastorino U, Buyse M, Friedel G, et al. Long-term results of lung metastasectomy: prognostic analyses based on 5206 cases. J Thorac Cardiovasc Surg 1997; 113:37-49

2 Pastorino U. The development of an international registry. I Thorac Oncol 2010;5(6 Suppl 2):S196-7.

3 Hellman S, Weichselbaum RR. Oligometastases. J Clin Oncol 1995;13:8-10.

4 Treasure T. Oligometastatic cancer: an entity, a useful concept, or a therapeutic opportunity? I R Soc Med 2012;105:242-6.

5 Weichselbaum RR, Hellman S. Oligometastases revisited. Nat Rev Clin Oncol 2011;8:378-82.

6 Sturgeon CM, Lai LC, Duffy MJ. Serum tumour markers: how to order and interpret them. BMJ 2009;339:b3527.

7 Pfannschmidt J, Zabeck H, Muley T, et al. Pulmonary metastasectomy following chemotherapy in patients with testicular tumors: experience in 52 patients. Thorac Cardiovasc Surg 2006:54:484-8.

8 Gonzalez $\mathrm{M}$, Poncet $\mathrm{A}$, Combescure $\mathrm{C}$, et al. Risk factors for survival after lung metastasectomy in colorectal cancer patients: a systematic review and meta-analysis. Ann Surg Oncol 2013:20:572-9.

9 Treasure T. Carcinoembryonic antigen: its place in decision making for pulmonary metastasectomy in colorectal cancer. J Thorac Oncol 2010;5(6 Suppl 2):S179-81.

10 Northover J, Houghton J, Lennon T. CEA to detect recurrence of colon cancer. JAMA 1994:272:31.

11 Pfannschmidt J, Hoffmann $\mathrm{H}$, Dienemann $\mathrm{H}$. Thoracic metastasectomy for nonseminomatous germ cell tumors. J Thorac Oncol 2010:5(6 Suppl 2):S182-6.
12 Besse B, Grunenwald D, Flechon A, et al. Nonseminomatous germ cell tumors: assessing the need for postchemotherapy contralateral pulmonary resection in patients with ipsilateral complete necrosis. J Thorac Cardiovasc Surg 2009;137:448-52.

13 Treasure T, Fiorentino F, Scarci M, et al. Pulmonary metastasectomy for sarcoma: a systematic review of reported outcomes in the context of Thames Cancer Registry data. BMJ Open 2012;2:5. doi:10.1136 bmjopen-2012-001736.

14 Kager L, Zoubek A, Potschger U, et al. Primary metastatic osteosarcoma: presentation and outcome of patients treated on neoadjuvant Cooperative Osteosarcoma Study Group protocols. I Clin Oncol 2003;21:2011-18.

15 Gelderblom H, Jinks RC, Sydes M, et al. Survival after recurrent osteosarcoma: data from 3 European Osteosarcoma Intergroup (EOI) randomized controlled trials. Eur J Cancer 2011;47:895-902.

16 National Institute for Health and Care Excellence (NICE). Guidance on Cancer Services. Improving Outcomes for People with Sarcoma. The Manual. The National Institute for Health and Care Excellence (NICE), 2006. http://www.nice.org.uk/ nicemedia/live/10903/28934/28934.pdf

17 Treasure T, Utley M. Surgical removal of asymptomatic pulmonary metastases: time for better evidence. BMJ 2013:346:21-3.

18 Van Raemdonck $D$, Friedel $G$. The European society of thoracic surgeons lung metastasectomy project. J Thorac Oncol 2010;5(6 Suppl 2):S127-9.

19 Oliaro A, Filosso PL, Bruna MC, et al. Pulmonary metastasectomy for melanoma. J Thorac Oncol 2010;5(6 Suppl 2):S187-91.

20 Tafra L, Dale PS, Wanek LA, et al. Resection and adjuvant immunotherapy for melanoma metastatic to the lung and thorax. I Thorac Cardiovasc Surg 1995:110:119-28.

21 Petersen RP, Hanish SI, Haney JC, et al. Improved survival with pulmonary metastasectomy: an analysis of 1720 patients with pulmonary metastatic melanoma. J Thorac Cardiovasc Surg 2007;133:104-10.

22 Treasure W. Diagnosis and Risk Management in Primary Care: words that count, numbers that speak. London: Radcliffe Publishing, 2011.

23 Fiorentino F, Hunt I, Teoh $\mathrm{K}$, et al. Pulmonary metastasectomy in colorectal cancer: a systematic review and quantitative synthesis. J $R$ Soc Med 2010;103:60-6.

24 Ollila DW, Stern SL, Morton DL. Tumor doubling time: a selection factor for pulmonary resection of metastatic melanoma. J Surg Oncol 1998;69:206-11.

25 Yang JC, Abad J, Sherry R. Treatment of oligometastases after successful immunotherapy. Semin Radiat Oncol 2006;16:131-5.

26 Fiorentino F, Vasilakis $C$, Treasure T. Clinical reports of pulmonary metastasectomy for colorectal cancer: a citation network analysis. Br J Cancer 2011;104:1085-97.

27 Embun R, Fiorentino F, Treasure T, et al. Pulmonary metastasectomy in colorectal cancer: a prospective study of demography and clinical characteristics of 543 patients in the Spanish colorectal metastasectomy registry (GECMP-CCR). BMJ Open 2013;3:e002787.

28 Treasure T, Utley M. Statistics for the rest of us: ten traps for the unwary in surgical series: a case study in mesothelioma reports. J Thorac Cardiovasc Surg 2007;133:1414-18.

29 Simms L, Barraclough H, Govindan R. Biostatistics primer: what a clinician ought to know-prognostic and predictive factors. J Thorac Oncol 2013;8:808-13.

30 Garcia-Yuste M, Cassivi S, Paleru C. Thoracic lymphatic involvement in patients having pulmonary metastasectomy: incidence and the effect on prognosis. J Thorac Oncol 2010:5(6 Suppl 2):S166-9.

31 Pfannschmidt J, Dienemann $\mathrm{H}$, Hoffmann $\mathrm{H}$. Surgical resection of pulmonary metastases from colorectal cancer: a systematic review of published series. Ann Thorac Surg 2007:84:324-38

32 Pfannschmidt J, Hoffmann H, Dienemann H. Reported outcome factors for pulmonary resection in metastatic colorectal cancer. J Thorac Oncol 2010;5(6 Suppl 2):S172-8

33 Utley $\mathrm{M}$, Treasure $\mathrm{T}$, Linklater $\mathrm{K}$, et al. Better out than in? The resection of pulmonary metastases from colorectal tumours. In: Xie X, Lorca F, Marcon E. eds. Operations Research for Health Care Engineering: Proceedings of the 33rd International Conference on Operational Research Applied to Health Services. Saint-Etienne: Publications de l'Universitaire de Saint-Etienne, 2008:493-500.

34 Aberg T, Malmberg KA, Nilsson B, et al. The effect of metastasectomy: fact or fiction? Ann Thorac Surg 1980;30:378-84.

35 Utley M, Treasure T. Interpreting data from surgical follow-up studies: the role of modeling. J Thorac Oncol 2010;5(6 Suppl 2):S200-2.

36 Levesque LE, Hanley JA, Kezouh A, et al. Problem of immortal time bias in cohort studies: example using statins for preventing progression of diabetes. BMJ 2010;340:b5087.

37 Suissa S. Immortal time bias in observational studies of drug effects. Pharmacoepidemiol Drug Saf 2007;16:241-9.

38 Jaklitsch MT, Mery CM, Lukanich JM, et al. Sequential thoracic metastasectomy prolongs survival by re-establishing local control within the chest. I Thorac Cardiovasc Surg 2001;121:657-67.

39 Burt BM, Ocejo S, Mery CM, et al. Repeated and aggressive pulmonary resections for leiomyosarcoma metastases extends survival. Ann Thorac Surg 2011;92:1202-7.

40 Bradford Hill A. The environment and disease: association or causation? Proc $R$ Soc Med 1965:58:295-300.

41 Glasziou P, Chalmers I, Rawlins M, et al. When are randomised trials unnecessary? Picking signal from noise. BMJ 2007:334:349-51. 